\title{
Particle and Blood Cell Dynamics in Oscillatory Flows Final Report
}

\author{
Juan M. Restrepo*(PI) \\ September 10, 2008
}

\section{Numerical Simulations of Forces Individual Particles}

\section{$1.1 \quad$ Goal}

Our aim has been to uncover fundamental aspects of the suspension and dislodgement of particles in wall-bounded oscillatory flows, in flows characterized by Reynolds numbers encompassing the situation found in rivers and near shores (and perhaps in some industrial processes). Our research tools are computational and our coverage of parameter space fairly broad. Computational means circumvent many complications that make the measurement of the dynamics of particles in a laboratory setting an impractical task, especially on the broad range of parameter space we plan to report upon.

The impact of this work on the geophysical problem of sedimentation is boosted considerably by the fact that the proposed calculations can be considered ab-initio, in the sense that little to no modeling is done in generating dynamics of the particles and of the moving fluid: we use a three-dimensional Navier Stokes solver along with straightforward boundary conditions. Hence, to the extent that Navier Stokes is a model for an ideal incompressible isotropic Newtonian fluid, the calculations yield benchmark values for such things as the drag, buoyancy, and lift of particles, in a highly controlled environment. Our approach will be to make measurements of the lift, drag, and buoyancy of particles, by considering progressively more complex physical configurations and physics.

\subsection{Activities Related to this Effort}

We produced three research articles and are presently working on a final one. In J. M. Restrepo, P. Fischer, G. K. Leaf "Forces on Particles in Oscillatory Boundary Layers", Journal of Fluid Mechanics, 468, pp. 327-347, 2002, we investigated the lift and drag forces, as a function of the Reynolds number, the Keulegan-Carpenter number. We succeeded in fully characterizing the lift and drag forces for all values of the parameters. Since the calculations used full Navier Stokes in three-dimensions and time, the data is fairly decisive with regard

*Mathematics Department and Physics Department, University of Arizona, Tucson, AZ 85721, USA. restrepo@math.arizona.edu 
to characterizing these forces: there is only numerical error and this, we demonstrated, is extremely small, when we compared data to actual experimental values of the forces (in a very limited regime of parameters). In this regard the paper succeeded in circumventing the nearly unsurmountable difficulties presented by the determination of the forces by experimental means.

Next, we computed the lift and drag forces in the situation whereby the particle is fixed a certain distance from the bounding wall. The work was succesfully completed and has been published (see P. Fischer, G. K. Leaf, J. M. Restrepo, "Influence of Wall Proximity on the Lift and Drag of a Particle in an Oscillatory Flow," Journal of Fluids Engineering, 127, pp. 583-594, 2005.). We obtained this way a comprehensive portrait of the lift and drag forces, as a function of the Reynolds number, the Keulegan-Carpenter number, and the gap between the particle and the hard wall. An important finding has been the discovery of a pressure-dominated and a viscous-dominated regime in the lift, as a function of the oscillatory frequency. Another important finding was the discovery that very few degrees of freedom are required to characterize the lift and drag fully in this situation.

The next step was to include rotation effects: Torque forces change the lift force dramatically via a Magnus effect in steady flows. The drag does not change. The question was whether this is the case if time dependent and oscillatory flows were affecting the particle. The findings were published in P. Fischer, G. Leaf, J. M. Restrepo, "Torque Effects on the Lift and Drag of Particles in an Oscillatory Boundary Flow," accepted, Journal of Fluids Engineering, pp 20+10 figures, May 2008. The lift, drag and torque for a particle fixed in space but allowed to rotate, subjected to an oscillatory/wall-bounded flow are thus mapped out with what could be considered experimental accuracy. Hence we know now the dependence of the particle lift, drag, and torque as a function of the Reynolds number, the Carpenter-Keulegan number, the gap number, and the buoyancy. We found that torque is indeed dramatically affected if the particle is forced to stay put. However, it becomes very weak if the particle is allowed to move. The lift/suction forces are most dramatically affected, whereas the drag is not. An important conclusion from this work is that torque, for a freely moving particle, is not crucial to the dynamics (this is an isolated particle, however), the lift force cannot be neglected, however, when the buoyancy forces are comparable to the lift force or smaller. Figure 1 shows the forces on a particle in a $R e=200$ flow.

Particle translation is the remaining aspect to explore and this portion of the work will consist of dislodgement and suspension calculations. We are presently setting up the computational code that will permit us characterize the particle forces in this situation. It may seem that this would require a significant change to our Navier Stokes solver; in fact, at first it may seem that one would be required to invoke some sort of ALE code or immersed boundary technique. However, we devised a simple alternative to this: we derive the Navier-Stokes equations in an accelerated reference frame, oscillating back and forth in concert with the flow motion. The upshot is that we simply have to modify the existing code for this virtual force. We hope to complete this project in a year. 

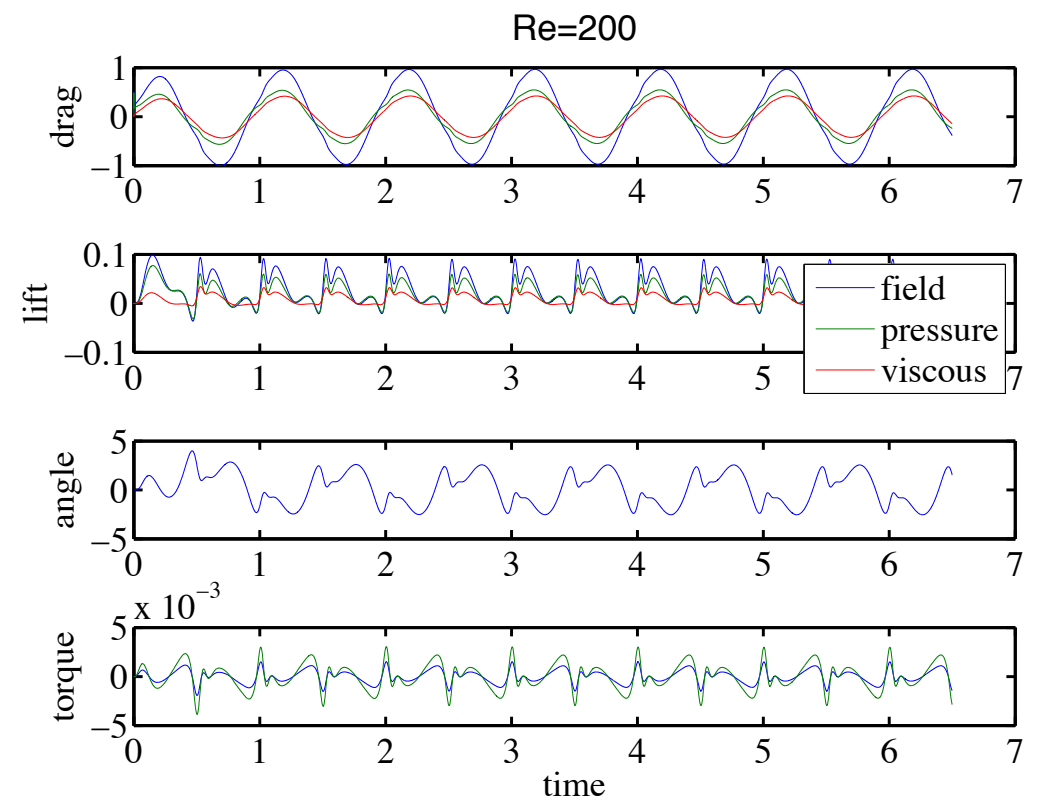

Figure 1: Forces on a particle in a wall-bounded oscillatory flow

\section{Shore-connected Sandbars}

Up-current rotated, shoreface-connected ridges are found in various coastal areas around the world. An often-quoted conjecture is that these ridges form during storm conditions through free instabilities in the erodible bed. Under these conditions both waves and currents are expected to play a significant roles in the hydrodynamics. Although some of the existing models referenced here have included the effects of waves parametrically in their bottom friction terms and sediment equations, the dynamical effects of wave-current interaction have not been explored. In the paper we begin to rectify this by considering the effects of wave-current interaction on the bed-form instabilities of a simple model. This brings up the possibility of unsteady longshore flows and questions about the roles of wave parameters and boundary conditions which we address here. We showed that the flow is stable under the wave forcing, however the waves do affect the bed-form instability. The main dynamical effect of the waves is in altering the shapes of the unstable modes. Under various conditions, however, waves may enhance or suppress the instability or introduce new unstable modes. They also affect the celerity of the ridges. In addition, we investigate the mechanisms whereby the waves affect the instability. We also show a potential problem with the parametrization in terms of wave orbital velocity.

This work appears in E. Lane, J. M. Restrepo, "Shoreface-connected Ridges under the Action of Currents and Waves," Journal of Fluid Mechanics, 582, DOI:0.1017/S0022112007005794 (2007). The growth rate and the shapes of the bars predicted appear in Figure 2 

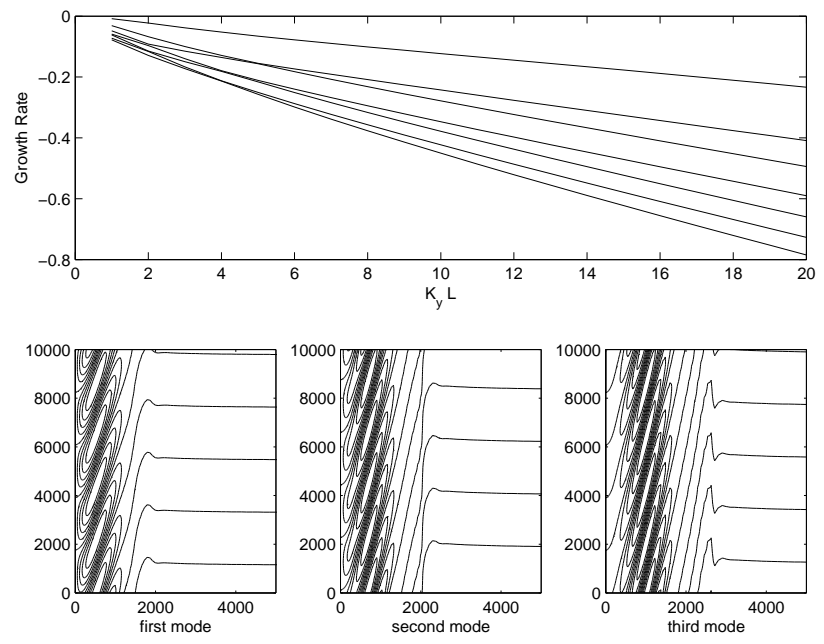

Figure 2: Shore-connected growth rate and eigenfunctions in a wave-current environment.

\section{Density Jets and Particle-laden Flows}

We considered the flow structure and stability of a planar saline jet descending into a stable density stratified fluid. The jet exhibits a rapid acceleration then deceleration as it encounters the more dense surrounding fluid. The entrained fluid from near the nozzle is dragged down then returns towards the nozzle forming a heart-shaped recirculation zone. The jet exhibits three instabilities: a symmetric instability in the jet core, an anti-symmetric instability in the jet core, and a symmetric instability along the edge of the entrained conduit. The neutral curves for the anti-symmetric instability are determined via direct numerical simulation.

The flow characteristics of a planar saline jet descending into a stable, density stratified fluid are significantly different than the flow profile of classical models jets and plumes. Unlike jets with monotonically decreasing centerline velocity or plumes with a monotonically increasing centerline velocity, the jet considered here accelerates the decelerates reaching a finite penetration length. In addition, a symmetric set of recirculation zones is formed along the jet where less dense water is entrained from near the nozzle, dragged down along the jet, then returned toward the nozzle as it encounters increasingly dense background fluid. This two-dimensional calculated flow profile is consistent with the experimental data and confirms the form of the theoretical velocity profile used in the analysis of the Hele-Shaw experiments. The jet appears in Figure 3

The jet exhibits three different instability modes: (1) an anti-symmetric mode in the jet core, that gives rise to the sinuous undulations observed in the Hele-Shaw experiments, (2) a symmetric mode in the jet core which results in a string of beads type of configuration, and (3) a symmetric set of waves along the edge of the jet conduit. All the modes may be unstable in the flow, although the anti-symmetric mode in the jet core will dominate the symmetric mode in the jet core. A local linear stability analysis confirms the presence of the three modes.

Overall, the flow becomes unstable for low Reynolds numbers at even relatively modest Grashof numbers. The characteristic return flow at the edge of the entrained conduit helps propagate a growing disturbance up and down the jet axis giving the instability its absolute 


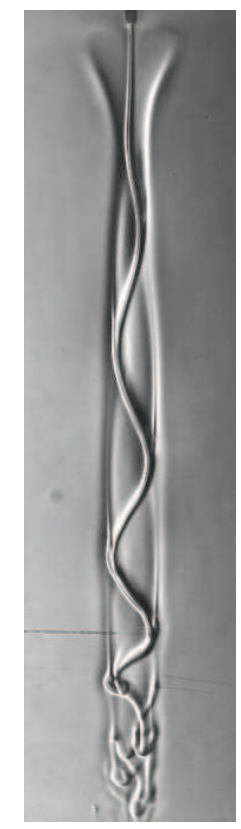

Figure 3: The density jet descending in a density-stratified fluid.

instability character. As would be expected, higher flux rates, corresponding to higher Reynolds numbers, decrease the stability of the flow. As the Schmidt number increases, the centerline velocity increases and the flow also becomes less stable. For sufficiently large

Schmidt numbers $(S c>500)$ the salt diffusion becomes negligible and has little influence on the flow stability. The effects of stratification length on the flow stability are much less clear. Longer stratification lengths allow the buoyant jet to accelerate more, increasing the velocity in the jet core. However, shorter stratification lengths lead to more rapid deceleration of the jet.

The work is currently under review in a paper by Sam Schofield and Juan M. Restrepo, "Stability of planar buoyant jets in stratified fluids" submitted to Physics of Fluids, May, 2008.

It is anticipated that the three dimensional case will exhibit similar stability characteristics with the anti-symmetric mode being replaced with a helical mode, and the symmetric mode in the jet core being replaced with an axisymmetric mode in the jet core. The three-dimensional experiments resulted in the following publication: C. Dombrowski, B. Lewellyn, A. I. Pesci, J. M. Restrepo, J. O. Kessler, and R. E. Goldstein, "Coiling, entrainment, and hydrodynamic coupling of decelerated fluid jets," Phys. Rev. Lett. 65, 184501 (2005).

In Figure 4 we show the experimental outcome from the sand ripple experiment. An explanation for the acceleration of the ripples in the steady flow is yet to be formulated. The dependence of the ripple height on the shearing forces appears in Figure 4b.

\section{An Unexpected Connection in Numerical Analysis}

An interesting and unexpected connection was made to numerical analysis: we required a quick and accurate estimate for the gap in the eigenvalue spectrum of a matrix (supposing 


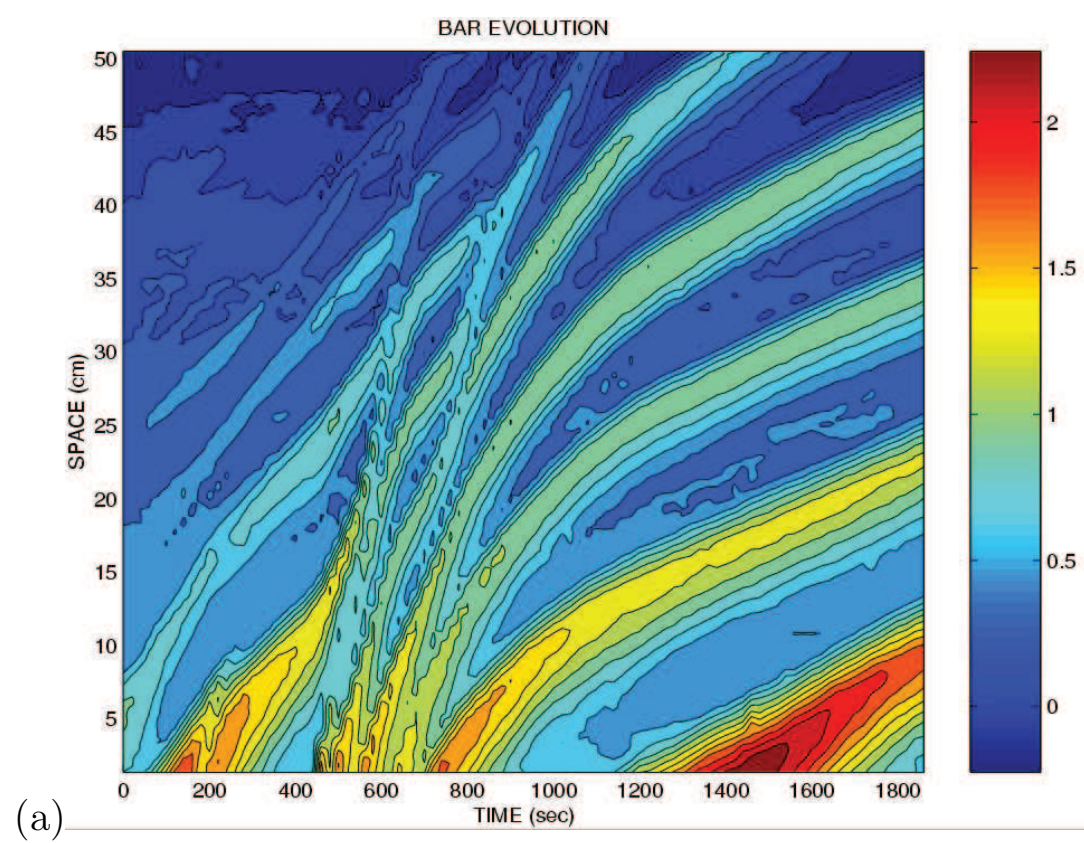

(b)

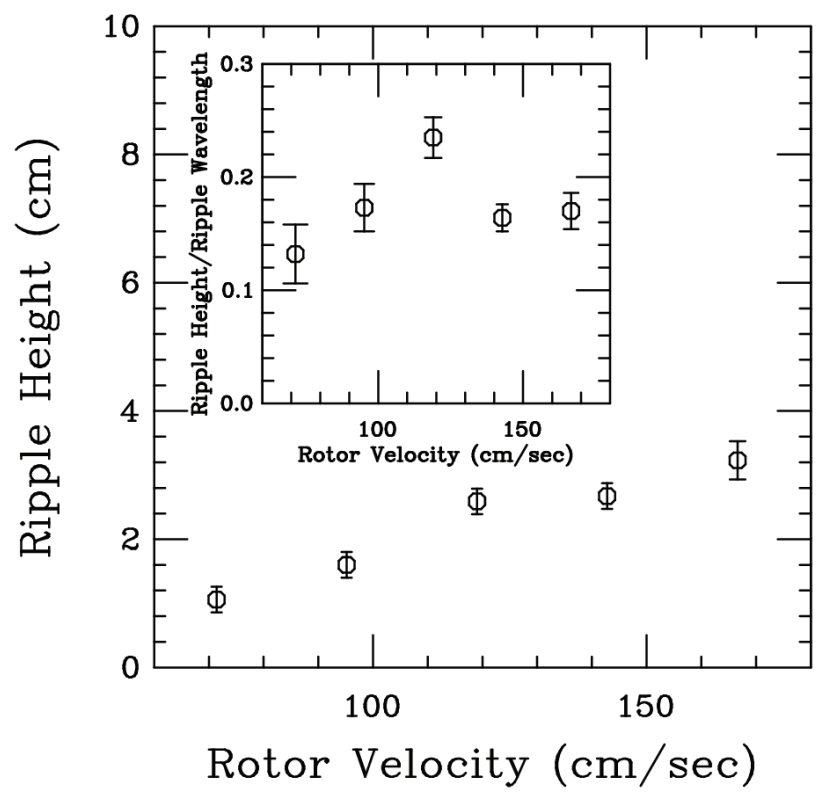

Figure 4: (a) Advection of sand ripples due to shearing forces. (b) Height dependence of the bars on the shearing strength 


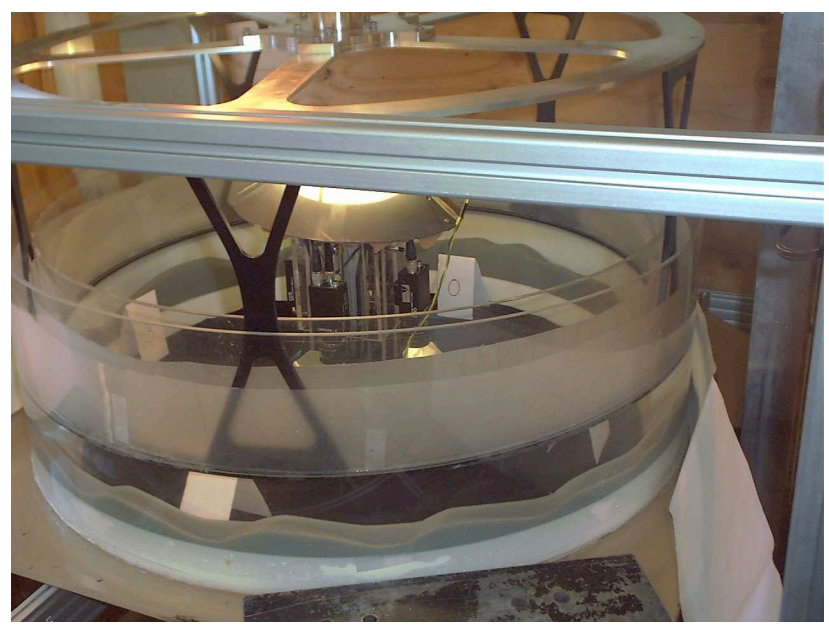

Figure 5: Ripple Tank

it existed) when computing the stability of the jets.

It was determined that to calculate all of the eigenvalues in order to find the gap was too expensive. With a post-doctoral fellow at U. Arizona we developed a scheme to detect the gap. In addition, we were able to use this information to build efficient preconditioners for linear algebra problems (this is totally unrelated to the original problem, but has been made part of the same project). The result are the papers M. Hasson, J.M. Restrepo, "Approximating on Disjoint Intervals and its Application to Matrix Preconditioning,", Complex Variables and Elliptic Equations, 52, DOI: 10.1080/17476930701524222 (2007) and the paper M. Hasson, J.M. Restrepo, J. M. Hyman, "A Strategy for Detecting Extreme Eigenvalues Bounding Gaps in the Discrete Spectrum of Self-Adjoint Operators," Computers and Mathematics with Applications, 53, pp. 1271-1283 (2007).

\section{$5 \quad$ Ripple Experiment}

In the ripple experiments we are looking at how millions of grains conspire to form bar structures when forced by a steady shearing fluid flow. This year has been mostly focused on improving significantly the apparatus. In a crude version of the experiment we have been able to determine that there are three fundamentally different evolutionary mechanisms for the erodible bed: an advective, a dispersive, and an explosive one. In the advective case the bars move along at a set precession velocity. The pattern changes little. In the dispersive case, a large lump of sand will resolve itself into an ordered set of sand piles downstream from the larger pile. In the explosive case the sand liquefies and moves as sheets at speeds significantly higher than the advective speed.

The apparatus appears in Figure 5

The outcomes from the experiment appear in J. M. Restrepo, H. Uys, R. E. Goldstein, A. I. Pesci, "Stably Precessive Granular Sand Bars Under Steady Shearing", under revision, July 2004, Physical Review Letters, pp $4+5$ figures. 


\section{Computational Blood Cell Dynamics}

Understanding the motion and deformation of red blood cells (RBCs) in the microvasculature can help scientists determine the distribution of RBCs, oxygen, and plasma-born solutes throughout the body. This project uses a two-dimensional computational model to consider isolated and multiple red blood cell motion through capillary branchings and in simple shear flows. The insights gained from these studies into how RBCs are distributed throughout the body can be used to help assess the efficacy of certain treatments which depend upon the distribution of solutes such as oxygen and drugs.

\subsection{Basic Mechanics}

Our two-dimensional model simulates the motion of the cross-section of a red blood cell as it moves through a given vessel geometry. A set of viscoelastic "external" elements (line segments) represent the elastic cytoskeleton and viscous lipid bilayer of the RBC membrane. Viscous "internal" elements represent the viscous cytoplasm and any out-of-plane membrane deformation effects. An internal pressure makes the cross-section resist changes in area. The cross-section is surrounded by a two-dimensional Stokes fluid (representing the plasma) that resides inside rigid vessel walls. The forces at each node (where line segments intersect, see Fig. 6) include fluid and internal pressure forces pushing on the membrane wall and forces coming from the internal and external elements including an additional bending elasticity. The sum of forces at each node is required to be zero. These "nodal equations" are coupled with the equations governing the surrounding Stokes flow. By solving this coupled system, instantaneous

velocities of cell nodes can be obtained and used to evolve the shape and position of the membrane inside arbitrary flow configurations.

\subsection{Computational Framework}

Given the instantaneous configuration of any cells in a given vessel bifurcation and appropriate boundary conditions, a finite element method program (FlexPDE version 5.0, PDE Solutions Inc., Antioch, CA) is used to solve the coupled system for the instantaneous velocities of any cells in the system. An explicit trapezoidal, order- 2 method uses these instantaneous velocities to advance the shape of the cell as it deforms and moves over time. When more than one cell is involved, the time step used in the explicit trapezoidal method changes so that the line segments representing the membranes of the cells do not cross over.

\subsection{Results}

Barber, JO, Alberding, JP, Restrepo, JM, Secomb, TW. Simulated Two-dimensional Red Blood Cell Motion, Deformation, and Partitioning in Microvessel Bifurcations. Ann. Biomed. Eng. 
While isolated RBCs follow the underlying fluid streamlines to first approximation, there are two significant deviations from these streamlines that may occur as they pass through diverging vessel bifurcations (blood flows from a mother branch into two daughter branches). The first deviation occurs because cell's migrate toward the mother vessel center line upstream of the bifurcation. The second deviation occurs when cells obstruct daughter branches and are pulled into the branch they obstruct. Additional simulations with rigid particles show cell migration causes RBCs to favor entrance into daughter branches with higher flow (relative to the other daughter branch) while cell obstruction causes RBCs to favor entrance into daughter branches with lower flow. In addition, the model produces predictions for RBC distribution functions at vessel bifurcations that agree remarkably well, quantitatively, with three-dimensional predictions. These results can be found in:

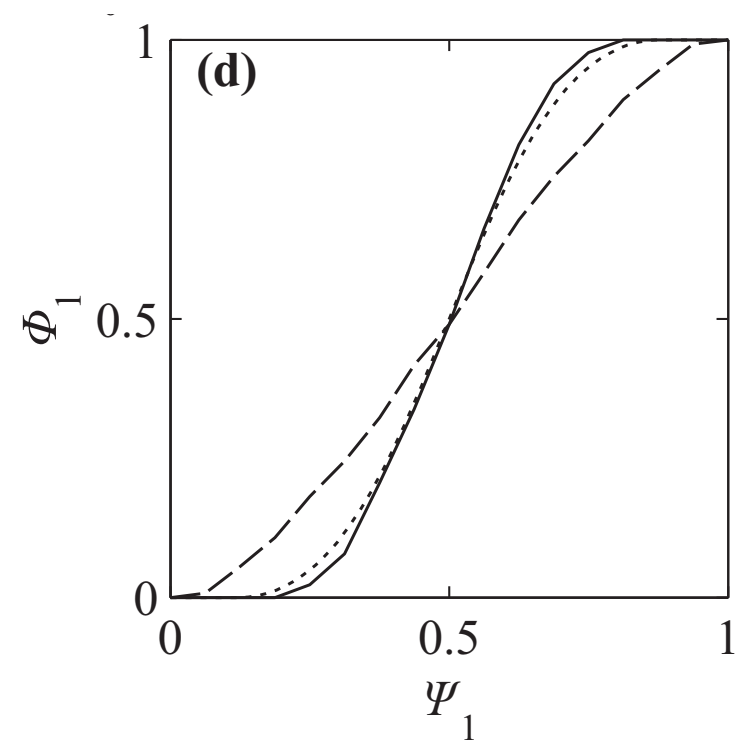

Figure 7: Fraction of red blood cells, $\Phi_{1}$, entering a daughter branch as a function of the fraction of plasma $\Psi_{1}$ entering the branch for the vessel bifurcation seen in Fig. 6.4 and very low hematocrits (one cell in bifurcation at a time). Experimental (dotted), flexible particle (solid), and rigid particle (dashed) estimates are included. Agreement between model and experiment is good.

Online First Available: doi:10.1007/s10439-008-9546-4.

The results were also presented at the following conferences:

- July 30-Aug 2, 2008. Simulated Two-Dimensional Red Blood Cell Motion, Deformation, and Partitioning in Microvessel Bifurcations. Society for Mathematical Biology 2008 Annual Meeting. (Talk.)

- July 7-11, 2008. Simulated Two-Dimensional RBC Motion, Deformation, and Partitioning in Microvessel Bifurcations. SIAM 2008 Annual Meeting. (Talk.)

- May 19-23, 2008. Red Blood Cell Motion in Bifurcating Microvessels. IPAM (UCLA) Optimal Transport Workshop IV: Optimal Transport in the Human Body: Lungs and Blood. (Poster.)

- August 15-19, 2007. Two-dimensional computational models of red blood cell motion through microvessel bifurcations and the effects of flexibility. 8th World Congress for the Microcirculation. (Poster.)

- February 9, 2007. Modeling Viscoelastic Red Blood Cell-like Membranes in Two Dimensions. University of Arizona, Los Alamos Days. (Talk.) 


\subsection{Present Work}

Currently the model is being employed to better understand multiple cell interaction as cells travel through a capillary bifurcation. It is also being used to study the diffusion of red blood cells in linear shear flows. Both studies enhance understanding of red blood cell distribution in the microvessels and the latter study additionally furthers understanding of diffusion of flexible particles in general flows.
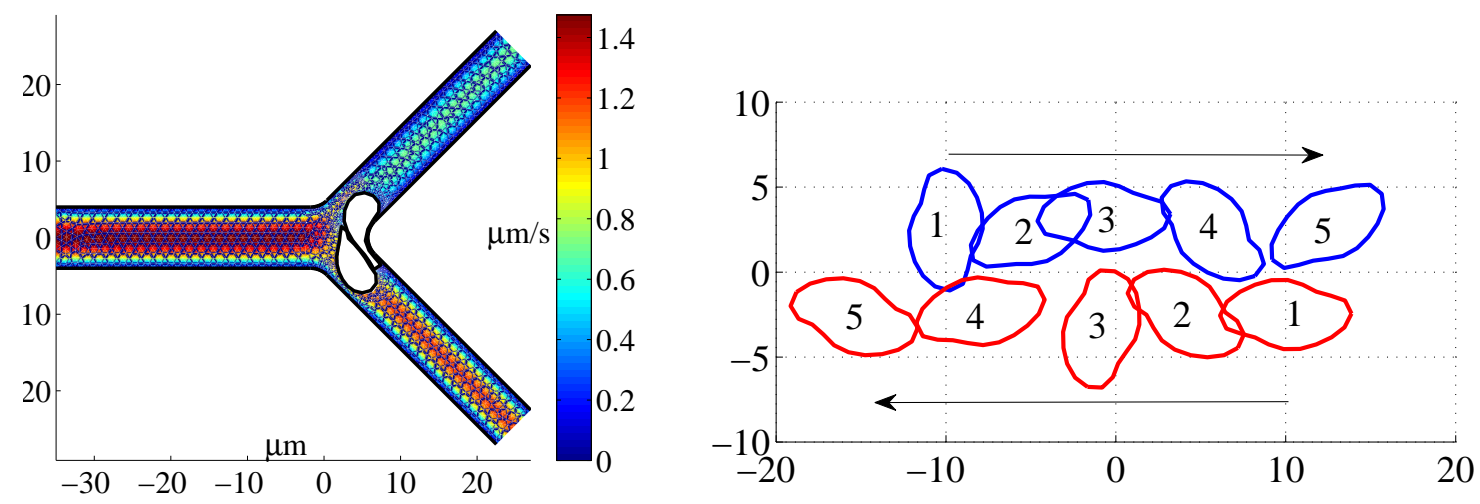

Figure 8: Left figure shows a snapshot of the motion of two RBCs through a symmetric capillary bifurcation. Color indicates the magnitude of the velocity vectors. Right figure shows two cells passing each other in a linear shear flow. Times of snapshots are: 1) 0ms, 2) $3.4 \mathrm{~ms}, 3) 5.9 \mathrm{~ms}, 4$ ) $9.4 \mathrm{~ms}$, and 5) $13.4 \mathrm{~ms}$.

\section{Education and Training}

The following are students whom were supported by the grant in some way.

- Sam Schofield, PhD

- Emily Lane, $\mathrm{PhD}$

- Yun Kang, MS

- Wendy Thomas, MS

- Brad Weir, presently ABD PhD

- Jared Barber, presently ABD PhD

- Maurice Hasson, Post-doc. 